\title{
Postoperative respiratory failure necessitating transfer to the intensive care unit in orthopedic surgery patients: risk factors, costs, and outcomes
}

\author{
Roman Melamed ${ }^{1 *}$, Lori L. Boland², James P. Normington², Rebecca M. Prenevost ${ }^{2}$, Lindsay Y. Hur ${ }^{3}$, \\ Leslie F. Maynard ${ }^{4}$, Molly A. McNaughton ${ }^{4}$, Tyler G. Kinzy ${ }^{2}$, Adnan Masood ${ }^{1}$, Mehdi Dastrange ${ }^{5}$ \\ and Joseph A. Huguelet ${ }^{5}$
}

\begin{abstract}
Background: Postoperative pulmonary complications in orthopedic surgery patients have been associated with worse clinical outcomes. Identifying patients with respiratory risk factors requiring enhanced monitoring and management modifications is an important part of postoperative care. Patients with unanticipated respiratory decompensation requiring transfer to the intensive care unit (ICU) have not been studied in sufficient detail.

Methods: A retrospective case-control study of elective orthopedic surgery patients (knee, hip, shoulder, or spine, $n=51$ ) who developed unanticipated respiratory failure (RF) necessitating transfer to the ICU over a 3-year period was conducted. Controls $(n=153)$ were frequency matched to cases by gender, age, and surgical procedure. Patient and perioperative care factors, clinical outcomes, and cost of care were examined.
\end{abstract}

Results: Transfer to the ICU occurred within 48 h of surgery in $73 \%$ of the cases, $31 \%$ required non-invasive ventilation, and $18 \%$ required mechanical ventilation. Cases had a higher prevalence of chronic obstructive pulmonary disease (COPD), obstructive sleep apnea (OSA), and regular psychotropic medication use than controls. Cases received more intravenous opioids during the first 24 postoperative hours, were hospitalized 4 days longer, had higher in-hospital mortality, and had excess hospitalization costs of US\$26,571. COPD, OSA, preoperative psychotropic medications, and anesthesia time were associated with risk of RF in a multivariate analysis.

Conclusions: Unanticipated RF after orthopedic surgery is associated with extended hospitalization, increased mortality, and higher cost of care. Hospital protocols that include risk factor assessment, enhanced monitoring, and a cautious approach to opioid use in high-risk patients may reduce the frequency of this complication.

Keywords: Respiratory failure, Orthopedic procedure, In-hospital, Critical care medicine, Intensive care unit transfer

\section{Background}

Demand for elective orthopedic procedures in the USA is expected to grow (Kurtz et al. 2007), and these procedures can be associated with serious cardiorespiratory complications (Dahl 1997). Identifying patients at high risk for postoperative pulmonary complications (PPC) and managing modifiable risk factors is essential, but

\footnotetext{
* Correspondence: roman.melamed@allina.com

'Department of Critical Care Medicine, Abbott Northwestern Hospital, 800 East 28th Street, Minneapolis, MN 55407, USA

Full list of author information is available at the end of the article
}

effective strategies are limited (Bapoje et al. 2007). PPC develop in 7-14 \% of patients undergoing spine or major joint surgery (Imposti et al. 2010; Lee et al. 2011; Lee et al. 2012; Lee et al. 2013; Ryu et al. 2010), and their occurrence is associated with longer length of stay and higher mortality (Lee et al. 2011; Lee et al. 2013; Lawrence et al. 2002; Liu et al. 2011; Smith et al. 2012). Potential PPC include pneumonia, atelectasis, pleural effusion, aspiration, airway compromise, acute respiratory distress syndrome, transfusion-related acute lung injury, fat or pulmonary embolism, opiate-related respiratory 
depression, and respiratory arrest (Imposti et al. 2010; Lee et al. 2012; Issack et al. 2009; Raw et al. 2003; Taylor and Gropper 2006), and their occurrence may be affected by the surgical site (Miura et al. 1996; Suk et al. 2006; Yoshida et al. 2007; Sagi et al. 2002).

Risk factors for PPC include advanced age, higher American Society of Anesthesiologists (ASA) class, smoking, chronic obstructive pulmonary disease (COPD), congestive heart failure (CHF), chronic renal failure, diabetes, and obstructive sleep apnea (OSA) (Imposti et al. 2010; Lee et al. 2012; Lee et al. 2013; Liu et al. 2011; Fu et al. 2011; Gupta et al. 2001; Memtsoudis et al. 2011; Mokhlesi et al. 2013; Moller et al. 2003; Roche et al. 2005; Weis et al. 1997). Intraoperative risk factors include surgical invasiveness, longer anesthesia time, thoracic-level procedure, and increased blood loss (Imposti et al. 2010; Lee et al. 2012; Sagi et al. 2002). The use of opioids in the postoperative period is also of concern as it may suppress central respiratory drive and cough and induce a monotonous respiratory pattern (Taylor and Gropper 2006), particularly in the elderly (Egbert 1996; Petre et al. 2012). However, studies of PPC have largely focused on preoperative and intraoperative predictors, and only one has quantified postoperative opioids in orthopedic surgery patients (Petre et al. 2012).

Estimated additional total costs related to PPC range from US $\$ 5983$ to US $\$ 120,579$ per procedure, with higher costs associated with mechanical ventilation or tracheostomy (Sabate et al. 2014). Opioid-related PPC are associated with increased lengths of stay and costs and are associated with higher opioid doses (Oderda et al. 2007).

We conducted a case-control study of orthopedic surgery patients who were transferred from the post-anesthesia care unit (PACU) to the general orthopedic floor but subsequently developed respiratory failure (RF) requiring transfer to the intensive care unit (ICU). The study objectives were to describe the causes of RF, to investigate potential perioperative factors which can cause postoperative deterioration, and to examine outcomes such as mortality and total cost of care in cases and controls.

\section{Methods}

We conducted a retrospective frequency-matched casecontrol study (1:3) among adult patients who underwent major orthopedic surgery between December 1, 2010, and November 30, 2013, at Abbott Northwestern Hospital, a tertiary hospital in Minneapolis, MN, that performs approximately 4000 spine and 2000 major joint surgeries annually. The study protocol was approved by the Schulman Institutional Review Board with a waiver of informed consent.

Cases were patients who underwent elective or semi-elective spine or major joint surgery and were postoperatively initially stable (i.e., oxygen saturation $>90 \%$ while receiving supplemental oxygen via nasal cannula and not requiring respiratory or hemodynamic support beyond standard care) but then experienced unanticipated RF and were subsequently transferred to the ICU. We queried the hospital's electronic health record (EHR) for procedural codes representing specific spine or major joint surgery based on the International Classification of Diseases 9th Revision (ICD-9). For descriptive purposes, patients were grouped by anatomical site of the surgery, i.e., knee, hip, spine (cervical), spine (non-cervical), and shoulder. We then restricted this list of patients to those who were initially stable and transferred directly from the PACU to a medical/surgical unit. Instances of unanticipated RF were then identified using a two-step process. First, we identified patients who were transferred to the ICU and had any of the following ICD-9 discharge diagnosis codes: 518.81 (acute RF), 518.84 (acute and chronic RF), 518.51 (acute RF following trauma/surgery), 518.52 (other pulmonary insufficiency following trauma/ surgery), 518.82 (acute pulmonary insufficiency), or 786.09 (respiratory distress). The medical records of these patients were then reviewed independently by two physicians ( $R M$ and $M D$ ) who confirmed RF if any of the following criteria were met: $\mathrm{pO}_{2}<60 \mathrm{mmHg}$ on room air or $\mathrm{pCO}_{2}>45 \mathrm{mmHg}$; increase in $\mathrm{pCO}_{2}$ from baseline or increase in supplemental oxygen requirements; or documentation of the patient's inability to protect airway or maintain adequate gas exchange. Controls were selected from among patients who underwent the same surgical procedures as the cases during the study timeframe but who were neither transferred to the ICU nor developed RF. Three controls were randomly selected for each case, frequency-matched on surgical procedure, gender, and 10year age category. Patients $<18$ years of age or who did not provide consent for use of their EHR data for research were excluded.

Patient demographics and event details including preoperative ASA class, length of stay, and in-hospital mortality were extracted from the EHR. ICU length of stay and ventilatory support details were extracted for cases. Two physicians (RM and MD) independently reviewed patient care records to determine the primary cause of RF; conditions present on admission such as COPD, OSA, and regular use of psychotropic medications (defined as antipsychotics, antidepressants, benzodiazepines, sleep aides, or muscle relaxants); and total operative anesthesia time (minutes). Total cost of care was obtained via financial databases maintained by the hospital.

Clinicians with expertise in pain management $(\mathrm{LYH}$, LFM, MAM) reviewed EHR data and quantified postoperative opioid use. Use of patient-controlled analgesia (PCA) was categorized as none, incremental dosing only, or a combination of a continuous basal 
rate and incremental dosing. Total morphine equivalents (milligrams) were computed for 12 consecutive 8 -h intervals from the end of surgery up to $96 \mathrm{~h}$ after surgery or patient discharge (whichever occurred sooner) using a standardized conversion tool developed by Allina Health Pharmacy Services based on established methods (McPherson 2009). Postoperative use of other sedatives such as diazepam and lorazepam was also documented.

\section{Analysis}

Continuous variables were described using means or medians based on results of Shapiro-Wilk tests for normality, and values in cases and controls were compared using $t$ tests or Wilcoxon-Mann-Whitney rank-sum tests accordingly. Categorical data were described using counts and proportions, with differences between cases and controls assessed using Fisher's exact test. Unconditional logistic regression models were used to estimate adjusted odds ratios and $95 \%$ confidence intervals for associations between hypothesized risk factors and RF. All analyses were conducted using Stata 12.1 (StataCorp LP, College Station, TX).

\section{Results}

Fifty-one cases of unanticipated postoperative RF occurred among 14,465 qualifying surgical procedures performed during the study timeframe $(0.4 \%)$. The most common procedure-related diagnoses were spinal stenosis and degenerative joint disease. There was no significant difference in obesity between cases and controls, but cases had a statistically significantly higher prevalence of COPD, OSA, chronic kidney disease (CKD), and psychotropic medication use and higher ASA class (Table 1). Among the 35 patients with diagnosed OSA, $35 \%$ of cases $(5 / 18)$ and $71 \%$ of controls $(n=12 / 17)$ were using continuous positive airway pressure (CPAP) at home at the time of admission $(p=0.02)$, and among 16 patients with COPD, $18 \%$ of cases (2/11) and $20 \%$ of controls $(1 / 5)$ were using home oxygen.

The most common primary causes of RF were decreased levels of consciousness with depressed respiratory drive (with or without laboratory confirmation of hypercarbia), pneumonia, CHF, and COPD exacerbation (Table 2). There were three cases of cardiac arrest: two related to respiratory depression and one due to acute myocardial infarction. Most cases (72 \%) developed RF within the first 2 postoperative days, with a median time from the end of surgery to the ICU transfer of $42 \mathrm{~h}$.

Spine and shoulder surgery patients received general anesthesia while regional anesthesia was used for knee and hip procedures. Cases generally had longer anesthesia times and higher estimated blood loss than controls, but there was no significant difference between the groups with regard to transfusion requirements (data not shown). Among spine surgery patients, cases received slightly higher doses of intraoperative opioids, but this difference was not observed in major joint surgery patients (Table 3).

Overall, cases received more intravenous opioids during the first 24 postoperative hours than controls (median 110 vs $73 \mathrm{mg}, p=0.006$ ), and opioid use was examined separately for patients with spine versus major joint procedures (Table 3 ). In spine surgery patients, the use of any PCA was more frequent in cases than in controls, and cases were more likely to have used a combination of continuous and incremental PCA than controls. Total postoperative opioids were generally higher in spine surgery patients than in major joint surgery patients. In major joint surgery patients, doses received in the first 8- and 24-h intervals were positively associated with the development of RF, with the median total dose in the first 8-h interval nearly three times higher in cases than in controls. Data in spine surgery patients suggest a similar pattern of higher opioid doses in cases across the 96-h postoperative interval. Use of additional sedatives during the postoperative course was associated with RF in spine surgery patients.

Among the cases and controls with previously diagnosed OSA, CPAP was started in the PACU in $11 \%$ of cases $(2 / 18)$ and $29 \%$ of controls $(5 / 17 ; p=0.23)$. Non-invasive ventilation (NIV) was ultimately initiated in $56 \%$ of cases who had OSA (10/18), commonly as a rescue therapy after the development of respiratory insufficiency.

Nine cases required mechanical ventilation (median hours $=56$ ), 16 were managed with non-invasive ventilation, and in-hospital mortality was $6 \%$ in cases and $0 \%$ in controls $(p=0.003$, Table 4$)$. Average hospitalization cost was significantly higher in cases (US\$46,456) than in controls $(\$ 19,885, p<0.001)$.

Multivariate logistic regression models initially included the three matching variables and the following covariates that were statistically significant in univariate analyses $(p \leq 0.01)$ : ASA class, COPD, OSA, CKD, smoking, psychotropic medication use, total operative anesthesia time, estimated blood loss, and opioids in the first 24 postoperative hours. Due to collinearity with comorbidities, ASA class was omitted from the final model, the results of which are presented in Table 5. COPD, OSA, CKD, psychotropic medication use, and total anesthesia time were all independently associated with risk of RF, but opioids in the first 24 postoperative hours were not associated with $\mathrm{RF}$ in the presence of these other factors.

\section{Discussion}

Previous studies of PPC have been conducted in more heterogeneous groups of surgical patients and have not 
Table 1 Surgical procedures, patient characteristics, and conditions present on admission

\begin{tabular}{lll}
\hline Variable & Cases $(n=51)$ & Controls \\
\hline $\begin{array}{l}\text { Surgical procedure type } \\
\text { Knee }\end{array}$ & $37 \%(19)$ & $37 \%(57)$ \\
Spine (cervical) & $41 \%(21)$ & $41 \%(63)$ \\
Spine (non-cervical) & $16 \%(8)$ & $16 \%(24)$ \\
Hip & $4 \%(2)$ & $4 \%(6)$ \\
Shoulder & $2 \%(1)$ & $2 \%(3)$ \\
Age, years & $66(34-88)$ & $67(32-90)$ \\
Age category, years & & \\
$31-40$ & $2 \%(1)$ & $2 \%(3)$ \\
$41-50$ & $6 \%(3)$ & $6 \%(9)$ \\
$51-60$ & $22 \%(11)$ & $22 \%(33)$ \\
$61-70$ & $33 \%(17)$ & $33 \%(51)$ \\
$71-80$ & $22 \%(11)$ & $22 \%(33)$ \\
$81+$ & $16 \%(8)$ & $16 \%(24)$ \\
Male & $35 \%(18)$ & $35 \%(54)$
\end{tabular}

Body mass index (BMI), $\mathrm{kg} / \mathrm{m}^{2}$

$\begin{array}{llll}<25 & 14 \%(7) & 17 \%(26) & 0.67 \\ 25.0-29.9 & 43 \%(22) & 37 \%(56) & 0.41 \\ 30.0-35.0 & 18 \%(9) & 25 \%(38) & 0.34 \\ >35.0 & 26 \%(13) & 22 \%(33) & 0.57 \\ \text { ASA class } & & & \\ 1 & 0 \%(0) & 2 \%(3) & 0.58 \\ 2 & 41 \%(21) & 69 \%(105) & <0.001 \\ 3 & 57 \%(29) & 29 \%(44) & <0.001 \\ 4 & 2 \%(1) & 1 \%(1) & 0.44\end{array}$

Conditions present

on admission

\begin{tabular}{|c|c|c|c|}
\hline Asthma & $20 \%(10)$ & $10 \%(16)$ & 0.10 \\
\hline COPD & $22 \%(11)$ & $3 \%(5)$ & $<0.001$ \\
\hline OSA & $35 \%(18)$ & $11 \%(17)$ & $<0.001$ \\
\hline Obesity (BMI $\geq 30 \mathrm{~kg} / \mathrm{m}^{2}$ ) & $43 \%(22)$ & $46 \%(71)$ & 0.75 \\
\hline $\mathrm{CHF}$ & $10 \%(5)$ & $2 \%(3)$ & 0.03 \\
\hline CKD & $22 \%(11)$ & $6 \%(9)$ & 0.002 \\
\hline Restrictive lung disease & $4 \%(2)$ & $1 \%(1)$ & 0.16 \\
\hline Opioid use & $41 \%(21)$ & $34 \%(52)$ & 0.40 \\
\hline Smoking history & $57 \%(29)$ & $37 \%(57)$ & 0.01 \\
\hline $\begin{array}{l}\text { Regular use of } \\
\text { psychotropic medication }\end{array}$ & $71 \%(36)$ & $37 \%(56)$ & $<0.001$ \\
\hline Diabetes & $18 \%(9)$ & $11 \%(17)$ & 0.23 \\
\hline
\end{tabular}

congestive heart failure, $C K D$ chronic kidney disease
Table 2 Cause of respiratory failure and timing of ICU transfer in cases

\begin{tabular}{ll}
\hline Variable & Cases $(n=51)$ \\
\hline Cause of respiratory failure & \\
Altered LOC with respiratory depression & $20 \%(10)$ \\
Hypercapnic respiratory failure & $18 \%(9)$ \\
Pneumonia & $16 \%(8)$ \\
CHF & $10 \%(5)$ \\
COPD exacerbation & $8 \%(4)$ \\
Aspiration & $6 \%(3)$ \\
Cardiac arrest & $6 \%(3)$ \\
Sepsis & $6 \%(3)$ \\
Pulmonary embolism & $4 \%(2)$ \\
Upper airway edema/obstruction & $4 \%(2)$ \\
Fat embolism & $2 \%(1)$ \\
OSA/OHS & $2 \%(1)$ \\
Total & $100 \%(51)$
\end{tabular}

Day transferred to ICU

$\begin{array}{ll}\text { Day } 0 & 12 \%(6) \\ \text { Day } 1 & 28 \%(14) \\ \text { Day } 2 & 33 \%(17) \\ \text { Days 3-4 } & 20 \%(10) \\ \text { Days 5-6 } & 8 \%(4) \\ \text { Time between end of surgery and transfer to ICU, hours } & 42(4-107)\end{array}$

Results are expressed as mean (range) or percent ( $n$ ) unless otherwise indicated ICU intensive care unit, $L O C$ loss of consciousness, CHF congestive heart failure, COPD chronic obstructive pulmonary disease, MI myocardial infarction, OSA obstructive sleep apnea, OHS obesity hypoventilation syndrome

distinguished between patients who were initially stable versus those who were directly transferred to the ICU after surgery. To our knowledge, this is the first casecontrol study of postoperative RF in orthopedic surgery patients to examine detailed information on postoperative opiate administration and to provide information on the primary causes of RF, clinical outcomes, and cost of care.

Postoperative RF is a significant complication in terms of increased mortality, length of stay, and cost (Dimick et al. 2004; Khuri et al. 2005), so there is strong interest in developing and validating tools that accurately identify high-risk patients for targeted intervention (Sabate et al. 2014; Canet and Gallart 2013). A scorecard quantifying the risk of opioid-related respiratory depression (Katie Felhofer 2013) and two scoring algorithms designed specifically for predicting postoperative RF have been proposed (Arozullah et al. 2000; Gupta et al. 2011). The demographic homogeneity of the surgical population that yielded one approach has been cited as a serious limitation (Arozullah et al. 2000), and while the score developed recently by Gupta et al. (Gupta et al. 
Table 3 Intraoperative and postoperative opioid use, by spine and major joint procedures

\begin{tabular}{|c|c|c|c|c|c|c|}
\hline \multirow{3}{*}{ Variable } & \multicolumn{3}{|l|}{ Spine surgery } & \multicolumn{3}{|c|}{ Major joint surgery } \\
\hline & \multirow{2}{*}{$\begin{array}{l}\text { Cases } \\
n=29\end{array}$} & \multirow{2}{*}{$\begin{array}{l}\text { Controls } \\
n=87\end{array}$} & \multirow[t]{2}{*}{$p$ value* } & \multirow{2}{*}{$\begin{array}{l}\text { Cases } \\
n=22\end{array}$} & \multirow{2}{*}{$\begin{array}{l}\text { Controls } \\
n=66\end{array}$} & \multirow[t]{2}{*}{$p$ value $^{*}$} \\
\hline & & & & & & \\
\hline Total intraoperative opioids (ME), mg & $115(95-185)$ & $85(69-120)$ & 0.006 & $18(15-45)$ & $23(15-68)$ & 0.77 \\
\hline \multicolumn{7}{|l|}{ Postoperative PCA } \\
\hline Incremental only & $21 \%(6)$ & $15 \%(13)$ & 0.56 & $0 \%(0)$ & $2 \%(1)$ & 1.00 \\
\hline Continuous and incremental & $69 \%(20)$ & $36 \%(31)$ & 0.002 & $0 \%(0)$ & $3 \%(2)$ & 1.00 \\
\hline None & $10 \%(3)$ & $49 \%(106)$ & $<0.001$ & $100 \%(22)$ & $95 \%(63)$ & 0.57 \\
\hline \multicolumn{7}{|l|}{ Total postoperative opioids (ME), mg } \\
\hline First $8 \mathrm{~h}$ & $40(0-470)$ & $33(0-179)$ & 0.42 & $26(0-107)$ & $9(0-240)$ & 0.01 \\
\hline First $24 \mathrm{~h}$ & $117(10-1414)$ & $87(0-448)$ & 0.05 & $109(15-224)$ & $64(0-499)$ & 0.03 \\
\hline First $48 \mathrm{~h}$ & $240(10-981)$ & $153(0-857)$ & 0.37 & $130(65-397)$ & $137(0-934)$ & 0.97 \\
\hline First $72 \mathrm{~h}$ & $416(20-1451)$ & $242(15-1262)$ & 0.33 & $174(112-236)$ & $190(17-1480)$ & 0.79 \\
\hline First $96 \mathrm{~h}$ & $716(408-1023)$ & $331(37-1667)$ & 0.64 & $112(112-112)$ & $189(22-2110)$ & 0.59 \\
\hline Use of other sedative during postoperative course & $83 \%(24)$ & $48 \%(42)$ & 0.001 & $64 \%(14)$ & $52 \%(34)$ & 0.46 \\
\hline
\end{tabular}

Results are expressed as median (range) or percent $(n)$ unless otherwise indicated $P C A$ patient-controlled analgesia, ME morphine equivalents

*Fisher's exact test or Wilcoxon rank-sum test as appropriate

2011) appears promising in terms of more generalizable use, only $4 \%$ of the patients underwent orthopedic procedures and their criteria for RF were stricter (i.e., patients requiring intubation or mechanical ventilation for $>48 \mathrm{~h}$ ). In addition, only one of the five criteria used in this score (i.e., ASA class) would have any significant variability in the patients studied here.

Consistent with previous studies, we found that risk of $\mathrm{RF}$ was associated with preexisting COPD, OSA, CHF, and CKD, as well as with smoking, longer anesthesia time, and higher intraoperative blood loss (Imposti et al.

Table 4 Hospitalization details and outcomes

\begin{tabular}{llll}
\hline Variable & Cases $(n=51)$ & Controls $(n=153)$ & $p$ value $^{*}$ \\
\hline Mechanical ventilation & $18 \%(9)$ & & \\
$\begin{array}{l}\text { Non-invasive ventilatory } \\
\text { support (BiPAP) }\end{array}$ & $31 \%(16)$ & & \\
$\begin{array}{l}\text { Total ventilation time, h } \\
\text { ICU length of stay, days }\end{array}$ & $1.6(1-347)$ & & \\
Hospital length of stay, days & $7(2-41)$ & $3(1-8)$ & $<0.001$ \\
In-hospital mortality & $6 \%(3)$ & $0 \%(0)$ & 0.003 \\
Cost of hospitalization, \$US & 46,456 & 19,885 & $<0.001$ \\
Discharge destination & & & \\
$\quad$ Died & $6 \%(3)$ & $0 \%(0)$ & 0.003 \\
Home & $55 \%(28)$ & $70 \%(107)$ & 0.046 \\
\multicolumn{1}{l}{ SNF/rehabilitation } & $39 \%(20)$ & $30 \%(46)$ & 0.193
\end{tabular}

Results are expressed as median (range) or percent $(n)$ unless otherwise indicated

ICU intensive care unit, SNF skilled nursing facility

*Wilcoxon rank-sum test, two-sample $t$ test, or Fisher's exact test, as appropriate

${ }^{\mathrm{a}}$ Mean US dollars
2010; Lee et al. 2011; Lee et al. 2012; Sagi et al. 2002; Gupta et al. 2001; Moller et al. 2003; Roche et al. 2005). Advanced age was a strong risk factor for PPC in previous studies (Imposti et al. 2010; Lee et al. 2012; Canet and Gallart 2013; Smetana et al. 2006), although we find it noteworthy that almost $30 \%$ of our cases were under the age of 60 .

OSA is a common disorder associated with increased morbidity and mortality (Peppard et al. 2013; Young et al. 2009). In a large analysis of orthopedic surgery patients, OSA was present in $8.4 \%$ and was associated with increased pulmonary and cardiac complications, use of intensive care, ventilatory support, and longer hospitalization (Memtsoudis et al. 2014). The postoperative state has been shown to exacerbate OSA symptoms and even cause sleep-disordered breathing in patients without OSA (Chung et al. 2014). In our study, cases with OSA had lower rates of compliance with home CPAP than controls. A validated OSA screening tool as described by Chung et al (Chung et al. 2016) could potentially be used to prompt earlier NIV use and enhanced postoperative monitoring in orthopedic surgical patients with diagnosed or suspected OSA.

Postoperative pain management with opioids presents significant challenges in orthopedic surgery patients (Taylor and Gropper 2006). Opioid-induced oversedation and associated respiratory depression is a principal concern, particularly in the presence of OSA, obesity, advanced age, preexisting pulmonary or cardiac disease, and longer anesthesia times (The Joint Commission 2012). But while opioid use may be an important risk factor for PPC, it is noticeably absent from the 
Table 5 Adjusted odds ratios and $95 \%$ confidence intervals of unplanned postoperative respiratory failure according to selected risk factors

\begin{tabular}{lll}
\hline Variable & OR & $95 \% \mathrm{Cl}$ \\
\hline COPD & 4.87 & $(1.24-19.21)$ \\
OSA & 4.79 & $(1.81-12.67)$ \\
CKD & 4.83 & $(1.36-17.17)$ \\
$\begin{array}{l}\text { Regular use of psychotropic } \\
\text { medication }\end{array}$ & 4.02 & $(1.58-10.26)$ \\
Total operative anesthesia time & 1.27 & $(1.10-1.48)$ \\
$\begin{array}{l}\text { Total opioid dose in the first } \\
\text { 24-h postoperative interval }\end{array}$ & 1.02 & $(0.98-1.06)$ \\
\hline
\end{tabular}

Results from a logistic regression model that included gender, surgical procedure type, age (10-year categories), and all variables shown in the table $O R$ odds ratio, $\mathrm{Cl}$ confidence interval, $C O P D$ chronic obstructive pulmonary disease, OSA obstructive sleep apnea, CKD chronic kidney disease

summarized evidence presented in two recent systematic reviews (Sabate et al. 2014; Canet and Gallart 2013). Data from the only other study that has quantified postoperative opioids when examining PPC in orthopedic surgery patients (Petre et al. 2012) suggest that higher doses of opioids may be associated with increased risk of PPC. In our study, cases had received significantly more opioids than controls in the first 8-24 postoperative hours and typically required transfer to the ICU within the first $48 \mathrm{~h}$ after surgery. The initial 24-48 postoperative hours thus may represent a particularly sensitive period when patients are prone to the development of respiratory insufficiency and the side effects of opioids, a theory that is supported by evidence that postoperative patients who experience analgesic-related respiratory events are more likely to have those events in the first $24 \mathrm{~h}$ after surgery (Taylor et al. 2005). Our finding that the use of additional sedatives during the postoperative course was also associated with RF further supports this hypothesis, but this topic requires further study.

Cases in our study remained hospitalized an average of 4 days longer than controls, and on average, total costs were US\$26,571 more for their hospitalization. Nearly one in five cases in our study required mechanical ventilation, and previous studies estimate that patients requiring intubation have longer hospital stays and higher total charges (Liu et al. 2011; Gupta et al. 2001; Mokhlesi et al. 2013).

Our results highlight the need for postoperative protocols that incorporate the type and complexity of the surgical procedure, perioperative events, individual patient risk factors, and facility-specific workflow and resources. Future studies should evaluate the effect on patient outcomes of clinical pathways that include patient assessment in the immediate postoperative period and a standardized hand-off process from anesthesia to the medical team. A mechanism for alerting the entire multidisciplinary team (e.g., nursing, hospitalists, respiratory care, pharmacists, surgeons) to the patient's risk factors and pain control, respiratory support, and monitoring needs would be an essential component of such protocols. This approach may reduce the frequency of both severe cases of RF requiring ICU transfer and respiratory events of lesser severity that can be managed outside the ICU.

Limitations of this study include the fact that we did not ascertain the entire spectrum of PPC in elective orthopedic surgery patients at our facility during the study timeframe. Second, given the single-center study design and narrowly defined patient population, our results may not be applicable to other treatment centers or surgical populations. Third, a detailed evaluation of the patient's preoperative cardiorespiratory and nutritional status was limited by the retrospective study design. Fourth, we were unable to blind the clinicians who computed total opioids from the case status of patients, but we do not believe this resulted in systematic differences in the assessment given the objective nature of the computation.

\section{Conclusions}

Unanticipated RF requiring ICU transfer occurs in less than $1 \%$ of patients who undergo elective orthopedic surgery, but despite their rarity, these events are associated with higher mortality, longer length of stay, and significantly increased hospital costs. Hospitals, and particularly high-volume orthopedic surgery centers, should consider a structured multidisciplinary team approach to postoperative management that involves systematic risk factor assessment, enhanced monitoring techniques, a judicious approach to pain management, and early identification and treatment of PPC.

\section{Abbreviations}

ASA class, American Society of Anesthesiologists physical status classification (system for assessing the fitness of patients before surgery); CHF, congestive heart failure; CKD, chronic kidney disease; COPD, chronic obstructive pulmonary disease; EHR, electronic health record; ICU, intensive care unit; LFM, Leslie F. Maynard, CNP (author); LYH, Lindsay Y. Hur, PharmD (author); MAM, Molly A. McNaughton, CNP (author); MD, Mehdi Dastrange, MD (author); OSA, obstructive sleep apnea; PACU, post-anesthesia care unit; PCA, patient-controlled analgesia; PPC, postoperative pulmonary complications; RF, respiratory failure; RM, Roman Melamed, MD (author)

\section{Authors' contributions}

All authors meet the criteria for authorship as recommended by the ICMJE. RM conceived of the study, participated in the study design and coordination, and helped to draft the manuscript. LB conceived of the study, performed the statistical analysis, participated in the study design and coordination, and helped to draft the manuscript. JN performed the statistical analysis, participated in the study design and coordination, and helped to draft the manuscript. RP performed the statistical analysis, participated in the study design, and helped to draft the manuscript. LH conceived of the study, participated in its design and coordination, and helped to draft the manuscript. LM conceived of the study, participated in its design and coordination, and helped to draft the manuscript. MM conceived of the study, participated in the study design and coordination, and helped to draft the manuscript. TK performed the statistical analysis, participated in the study design and coordination, and helped to draft the manuscript. AM conceived of the study, participated in the study design and 
coordination, and helped to draft the manuscript. MD conceived of the study, participated in the study design and coordination, and helped to draft the manuscript. JH participated in the study design and coordination and helped to draft the manuscript. All authors read and approved the final manuscript.

\section{Competing interests}

The authors declare that they have no competing interests.

\section{Ethics approval and consent to participate}

The study protocol was approved by the Schulman Institutional Review Board with a waiver of informed consent.

\section{Author details}

${ }^{1}$ Department of Critical Care Medicine, Abbott Northwestern Hospital, 800 East 28th Street, Minneapolis, MN 55407, USA. Division of Applied Research, Allina Health, 2925 Chicago Ave South, Minneapolis, MN, USA. ${ }^{3}$ Department of Pharmacy, Abbott Northwestern Hospital, 800 East 28th Street, Minneapolis, MN, USA. ${ }^{4}$ Chronic Pain Team, Abbott Northwestern Hospital, 800 East 28th Street, Minneapolis, MN, USA. ${ }^{5}$ Internal Medicine Residency Program, Abbott Northwestern Hospital, 800 East 28th Street, Minneapolis, MN, USA.

Received: 18 February 2016 Accepted: 1 July 2016

Published online: 02 August 2016

\section{References}

Arozullah AM, Daley J, Henderson WG, et al. Multifactorial risk index for predicting postoperative respiratory failure in men after major noncardiac surgery. The National Veterans Administration Surgical Quality Improvement Program. Ann Surg. 2000;232:242-53.

Bapoje SR, Whitaker JF, Schulz T, et al. Preoperative evaluation of the patient with pulmonary disease. Chest. 2007;132:1637-45.

Canet J, Gallart L. Predicting postoperative pulmonary complications in the general population. Curr Opin Anaesthesiol. 2013;26:107-15.

Chung F, Liao P, Yegneswaran B, et al. Postoperative changes in sleep-disordered breathing and sleep architecture in patients with obstructive sleep apnea. Anesthesiology. 2014;120:287-98.

Chung F, Abdullah HR, Liao P. STOP-Bang Questionnaire: a practical approach to screen for obstructive sleep apnea. Chest. 2016;149:631-8.

Dahl OE. Cardiorespiratory and vascular dysfunction related to major reconstructive orthopedic surgery. Acta Orthop Scand. 1997;68:607-14

Dimick JB, Chen SL, Taheri PA, et al. Hospital costs associated with surgical complications: a report from the private-sector National Surgical Quality Improvement Program. J Am Coll Surg. 2004;199:531-7.

Egbert AM. Postoperative pain management in the frail elderly. Clin Geriatr Med. 1996;12:583-99.

Fu KM, Smith JS, Polly Jr DW, et al. Correlation of higher preoperative American Society of Anesthesiology grade and increased morbidity and mortality rates in patients undergoing spine surgery. J Neurosurg Spine. 2011;14:470-4.

Gupta RM, Parvizi J, Hanssen AD, et al. Postoperative complications in patients with obstructive sleep apnea syndrome undergoing hip or knee replacement: a case-control study. Mayo Clin Proc. 2001;76:897-905.

Gupta H, Gupta PK, Fang X, et al. Development and validation of a risk calculator predicting postoperative respiratory failure. Chest. 2011;140:1207-15.

Imposti F, Cizik A, Bransford R, et al. Risk factors for pulmonary complications after spine surgery. Evid Based Spine Care J. 2010;1:26-33.

Issack PS, Lauerman MH, Helfet DL, et al. Fat embolism and respiratory distress associated with cemented femoral arthroplasty. Am J Orthop (Belle Mead NJ). 2009;38:72-6.

Katie Felhofer PD. Developing a respiratory depression scorecard for capnography monitoring. Innovations in Pharmacy. 2013;4:11.

Khuri SF, Henderson WG, DePalma RG, et al. Determinants of long-term survival after major surgery and the adverse effect of postoperative complications. Ann Surg. 2005;242:326-41. discussion 341-323.

Kurtz S, Ong K, Lau E, et al. Projections of primary and revision hip and knee arthroplasty in the United States from 2005 to 2030. J Bone Joint Surg Am. 2007;89:780-5

Lawrence VA, Hilsenbeck SG, Noveck H, et al. Medical complications and outcomes after hip fracture repair. Arch Intern Med. 2002;162:2053-7.
Lee MJ, Hacquebord J, Varshney A, et al. Risk factors for medical complication after lumbar spine surgery: a multivariate analysis of 767 patients. Spine (Phila Pa 1976). 2011;36:1801-6.

Lee MJ, Konodi MA, Cizik AM, et al. Risk factors for medical complication after spine surgery: a multivariate analysis of 1,591 patients. Spine J. 2012;12:197-206.

Lee MJ, Konodi MA, Cizik AM, et al. Risk factors for medical complication after cervical spine surgery: a multivariate analysis of 582 patients. Spine (Phila Pa 1976). 2013;38:223-8.

Liu SS, Chisholm MF, Ngeow J, et al. Postoperative hypoxemia in orthopedic patients with obstructive sleep apnea. HSS J. 2011;7:2-8.

McPherson ML. Demystifying opioid conversion calculations: a guide to effective dosing. 2009.

Memtsoudis S, Liu SS, Ma Y, et al. Perioperative pulmonary outcomes in patients with sleep apnea after noncardiac surgery. Anesth Analg. 2011;112:113-21.

Memtsoudis SG, Stundner O, Rasul R, et al. The impact of sleep apnea on postoperative utilization of resources and adverse outcomes. Anesth Analg. 2014;118:407-18.

Miura Y, Mimatsu K, Iwata H. Massive tongue swelling as a complication after spinal surgery. J Spinal Disord. 1996;9:339-41.

Mokhlesi B, Hovda MD, Vekhter B, et al. Sleep-disordered breathing and postoperative outcomes after elective surgery: analysis of the nationwide inpatient sample. Chest. 2013:144:903-14.

Moller AM, Pedersen T, Villebro N, et al. Effect of smoking on early complications after elective orthopaedic surgery. J Bone Joint Surg (Br). 2003;85:178-81.

Oderda GM, Said Q, Evans RS, et al. Opioid-related adverse drug events in surgical hospitalizations: impact on costs and length of stay. Ann Pharmacother. 2007:41:400-6.

Peppard PE, Young T, Barnet JH, et al. Increased prevalence of sleep-disordered breathing in adults. Am J Epidemiol. 2013;177:1006-14.

Petre BM, Roxbury CR, McCallum JR, et al. Pain reporting, opiate dosing, and the adverse effects of opiates after hip or knee replacement in patients 60 years old or older. Geriatr Orthop Surg Rehabil. 2012;3:3-7.

Raw DA, Beattie JK, Hunter JM. Anaesthesia for spinal surgery in adults. Br J Anaesth. 2003;91:886-904

Roche JJ, Wenn RT, Sahota O, et al. Effect of comorbidities and postoperative complications on mortality after hip fracture in elderly people: prospective observational cohort study. BMJ. 2005;331:1374.

Ryu YJ, Chun EM, Shim SS, et al. Risk factors for pulmonary complications, including pulmonary embolism, after total knee arthroplasty (TKA) in elderly Koreans. Arch Gerontol Geriatr. 2010;51:299-303.

Sabate S, Mazo V, Canet J. Predicting postoperative pulmonary complications: implications for outcomes and costs. Curr Opin Anaesthesiol. 2014;27:201-9.

Sagi HC, Beutler W, Carroll E, et al. Airway complications associated with surgery on the anterior cervical spine. Spine (Phila Pa 1976). 2002;27:949-53.

Smetana GW, Lawrence VA, Cornell JE, et al. Preoperative pulmonary risk stratification for noncardiothoracic surgery: systematic review for the American College of Physicians. Ann Intern Med. 2006;144:581-95.

Smith JS, Saulle D, Chen CJ, et al. Rates and causes of mortality associated with spine surgery based on 108,419 procedures: a review of the Scoliosis Research Society Morbidity and Mortality Database. Spine (Phila Pa 1976). 2012;37:1975-82

Suk KS, Kim KT, Lee SH, et al. Prevertebral soft tissue swelling after anterior cervical discectomy and fusion with plate fixation. Int Orthop. 2006:30:290-4.

Taylor JM, Gropper MA. Critical care challenges in orthopedic surgery patients. Crit Care Med. 2006;34:S191-199.

Taylor S, Kirton OC, Staff I, et al. Postoperative day one: a high risk period for respiratory events. Am J Surg. 2005;190:752-6.

The Joint Commission: safe use of opioids in hospitals. Sentinel Event Alert. 2012;1:5.

Weis JC, Betz RR, Clements 3rd DH, et al. Prevalence of perioperative complications after anterior spinal fusion for patients with idiopathic scoliosis. J Spinal Disord. 1997:10:371-5.

Yoshida M, Neo M, Fujibayashi S, et al. Upper-airway obstruction after short posterior occipitocervical fusion in a flexed position. Spine (Phila Pa 1976). 2007;32:E267-270.

Young T, Palta M, Dempsey J, et al. Burden of sleep apnea: rationale, design, and major findings of the Wisconsin Sleep Cohort study. WMJ. 2009;108:246-9. 